\title{
A numerical scheme for continuous population models for single and interacting species
}

\author{
Yalçın ÖZTÜRK ${ }^{1, *}$, Ayşe ANAPALI², Mustafa GÜLSU² \\ ${ }^{1}$ Ula Ali Koçman Vocational School, Muğla Sitkı Koçman University, Muğla, Turkey \\ ${ }^{2}$ Department of Mathematics, Faculty of Science, Muğla Sitkı Koçman University, Muğla, Turkey \\ Gelis Tarihi (Recived Date): 23.03.2016 \\ Kabul Tarihi (Accepted Date): 02.01.2017
}

\begin{abstract}
In this article, the dynamic of models such as logistic growth model, prey-predator model and 2-species Lotka-Volterra competition model is approximately solved by the Chebyshev collocation method. These nonlinear mathematical models are transformed into the matrix form by Chebyshev expansion method and converted nonlinear algebraic equation system. Chebyshev coefficients are obtained by solving nonlinear equation system. Results are compared with Homotopy perturbation and Adomian decomposition method and then comparision numerical result and exact solution are presented by graphics for logistic growth model. Plots are showed the numbers of prey and predator versus time for various $N$ values on predaor prey model. In the 2 spices Lotka Volterra competition model numerical results are presented by graphics. Matlab R2010a and Mapple14 are used for all calculations and graphs. In the conclusion part, the CPU times of the programs are given and the models are compared
\end{abstract}

Keywords: Logistic growth model, prey and predator model, Lotka-Volterra model, 2species Lotka-Volterra model, system of nonlinear differential equations

\section{Tek ve etkileşimli türlerin sürekli populasyon modelleri için bir sayısal yöntem}

\section{Özet}

Bu makalede, lojistik büyüme modeli, av avcı modeli ve 2-tür Lotka-Volterra yaşama mücadelesi modeli gibi modeller Chebyshev stralama metodu ile çözülmüştür. Bu lineer olmayan matematiksel modeller Chebyshev açılımı metodu ile matris formuna

\footnotetext{
*Yalçın ÖZTÜRK, yozturk@mu.edu.tr, http://orcid.org/0000-0002-4142-5633

Ayşe ANAPALI, ayseanapali@mu.edu.tr, http://orcid.org/0000-0002-0198-1862

Mustafa GÜLSU, mgulsu@mu.edu.tr, http://orcid.org/0000-0001-6139-0266
} 
dönüştürülmüş ve lineer olmayan cebirsel denklem sistemine indirgenmiştir. Lineer olmayan denklem sistemi çözülerek Chebyshev katsayıları elde edilmiştir. Lojistik büyüme modeli için sonuçlar homotopy perturbation metodu ve Adomian decomposition metodu ile karşılaştırılmış ve elde edilen nümerik sonuçlar ile tam çözümün karşılaştırılması grafiklerle sunulmuştur. Av-avcı modelinde grafikler yardımı ile av ve avcı sayılarının zamana karşı olan durumları farklı $N$ değerleri için gösterilmiştir. 2 tür Lotka Volterra yaşama mücadelesi modelinde nümerik sonuçlar grafik ile ifade edilmiştir. Yapılan tüm hesaplamalar ve grafik çizimlerinde Matlab R2010a ve Maple14 kullanılmıştır. Ayrıca sonuç kısmında programların CPU zamanları verilerek modeller arası karşılaştırmalar yapılmıştır.

Anahtar Kelimeler: Lojistik büyüme modeli, av-avcı modeli, Lotka-Volterra modeli, 2tür Lotka-Volterra modeli, linear olmayan diferansiyel denklem sistemi

\section{Introduction}

Modelling of ecological systems has received a great deal of attention from theoretical ecologists in the last few decades. Much focus has been on mathematical models of these systems, since they have substantially contributed to the understanding of the dynamics of systems by forging strong links between models and available data. Three of the most populer mathematical models are a logistic growth in population, a preypredator model: Lotka-Volterra system and a simple 2-species Lotka-Volterra competition model [1-12].

First, dynamic of the logistic growth of population as a single species is described the following equation [1]:

$$
\frac{d N}{d x}=r N\left(1-\frac{N}{K}\right)
$$

where $r$ and $K$ are positive constant. Here $N=N(x)$ is the population of the species at time $t$. In addition, $r(1-N / K)$ is the per capita growth rate and $K$ is carrying capacity of the environment. For simplifying the notations more,

$$
y(t)=\frac{N(t)}{K} \text { and } t=r x
$$

and its become

$$
\frac{d y}{d t}=y(1-y)
$$

If $N(0)=N_{0}$, then $y(0)=N_{0} / K$. Hence, the analytic solution of Eq.(2) is

$$
y(t)=\frac{1}{1+\left(K / N_{0}-1\right) e^{-t}}
$$


Second model is the predator-prey model: Lotka-Voleterra system as an interacting species [3-10]

$$
\begin{aligned}
& \frac{d N}{d t}=N(a-b P) \\
& \frac{d P}{d t}=P(c N-d)
\end{aligned}
$$

where $a, b, c$ and $d$ are constants and $N(t), P(t)$ are the populations of rabbits and the foxes at the time $t$ with initially rabbits and foxes populations are $N(0)=\alpha$, $P(0)=\beta$ respectively. In this model some rabbits and foxes live together. Foxes eat the rabbits and rabbits eat clover. Suppose that there are enough clovers and the rabbits have enough food to eat. There is an increase and decrease in the number of foxes and rabbits.

Third, we consider the simple 2-species Lotka-Volterra competition model. In this model each species $N_{1}$ and $N_{2}$ have logistic growth in the absence of the other $[1,11,12]$. The dynamic of model as

$$
\begin{aligned}
& \frac{d N_{2}}{d x}=r_{1} N_{1}\left(1-\frac{N_{1}}{K_{1}}-b_{12} \frac{N_{2}}{K_{1}}\right) \\
& \frac{d N_{2}}{d x}=r_{2} N_{2}\left(1-\frac{N_{2}}{K_{2}}-b_{21} \frac{N_{1}}{K_{2}}\right)
\end{aligned}
$$

where $r_{1}$ and $r_{2}$ are the linear birth rates and the $K_{1}$ and $K_{2}$ are carrying capacities. $b_{12}$ and $b_{22}$ measure the competitive effect of $N_{2}$ on $N_{1}$ and $N_{1}$ on $N_{2}$ recpectively(they are generally not equal). If we non-dimensionalize this model by writting

$$
u(t)=\frac{N_{1}(x)}{K_{1}}, v(t)=\frac{N_{2}(x)}{K_{2}}, x=r_{1} t, \rho=\frac{r_{2}}{r_{1}}, a=b_{12} \frac{K_{2}}{K_{1}}, b=b_{21} \frac{K_{1}}{K_{2}}
$$

the system given by Eq.(6)becomes [11-12]

$$
\begin{aligned}
& \frac{d u}{d t}=u(1-u-a v) \\
& \frac{d v}{d t}=\rho v(1-v-b u)
\end{aligned}
$$

In recently years, Chebyshev expansion method using for solving linear Fredholm, Volterra integro-differential difference equation [13-15], nonlinear differential equation such as Abel equation [16-17], systems of integral equations [18-19] and some numerical models [20-22]. We construct to the shifted Chebyshev series solutions that is,

$$
y_{N}(t)=\sum_{n=0}^{N} a_{n} T_{n}^{*}(t)
$$


where $T_{n}^{*}(t)$ denotes the shifted Chebyshev polynomials of the first kind, $a_{n}(0 \leq n \leq N)$ are unknown Chebyshev coefficients, and $\mathrm{N}$ is chosen any positive integer such that $N \geq m$.

\section{Chebyshev polynomials}

The Chebyshev polynomials $T_{n}(t)$ of the first kind is a polynomials in $x$ of degree $n$, defined by relation [23-25]

$$
T_{n}(t)=\cos n \theta, \text { when } t=\cos \theta
$$

If the range of the variable $x$ is the interval $[-1,1]$, the range the corresponding variables $\theta$ can be taken $[0, \pi]$. We map the independent variable $t$ in $[0,1]$ to the variable $s$ in $[-1,1]$ by transformation

$$
s=2 t-1 \text { or } t=\frac{1}{2}(s+1)
$$

and this lead to the shifted Chebyshev polynomial of the first kind $T_{n}^{*}(t)$ of degree $n$ in $x$ on [0,1] given by [23]

$$
T_{n}^{*}(t)=T_{n}(s)=T_{n}(2 t-1)
$$

These polynomials have the following properties [23-25]:

i) $T^{*}{ }_{n+1}(t)$ has exactly $n+1$ real zeroes on the interval $[0,1]$. The $i$-th zero $t_{i}$ is

$t_{i}=\frac{1}{2}\left(1+\cos \left(\frac{(2(n-i)+1) \pi}{2(n+1)}\right)\right), i=0,1, \ldots, n$

ii) It is well known that the relation between the powers of $t^{n}$ and the shifted Chebyshev polynomials $T_{n}^{*}(t)$ is

$t^{n}=2^{-2 n+1} \sum_{k=0}^{n} \cdot\left(\begin{array}{l}2 n \\ k\end{array}\right) T_{n-k}^{*}(t), 0 \leq x \leq 1$

where $\sum$ ' denotes a sum whose first term is halved.

\section{Fundamental relation}

Let us consider Eq. (1) and find the matrix forms of the equation. First we can convert the solution $y(t)$ defined by a truncated shifted Chebyshev series (3) and its derivative $y^{(k)}(t)$ to matrix forms

$$
y_{N}(t)=\mathbf{T}^{*}(t) \mathbf{A}, y_{N}^{(k)}(t)=\mathbf{T}^{*(k)}(t) \mathbf{A}, k=0,1,2
$$

where 


$$
\begin{aligned}
\mathbf{T}^{*}(t) & =\left[\begin{array}{lll}
T_{0}^{*}(t) & T_{1}^{*}(t) \ldots T_{N}^{*}(t)
\end{array}\right] \\
\mathbf{A} & =\left[\begin{array}{llll}
a_{0} & a_{1} \ldots & a_{N}
\end{array}\right]^{T}
\end{aligned}
$$

By using the expression (6) and taking $n=0,1, \ldots, N$ we find the corresponding matrix relation as follows

$$
(\mathbf{X}(t))^{T}=\mathbf{D}\left(\mathbf{T}^{*}(t)\right)^{T} \quad \text { and } \quad \mathbf{X}(t)=\mathbf{T}^{*}(t) \mathbf{D}^{T}
$$

where

$$
\begin{aligned}
& \mathbf{X}(t)=\left[1 t \ldots t^{N}\right]
\end{aligned}
$$

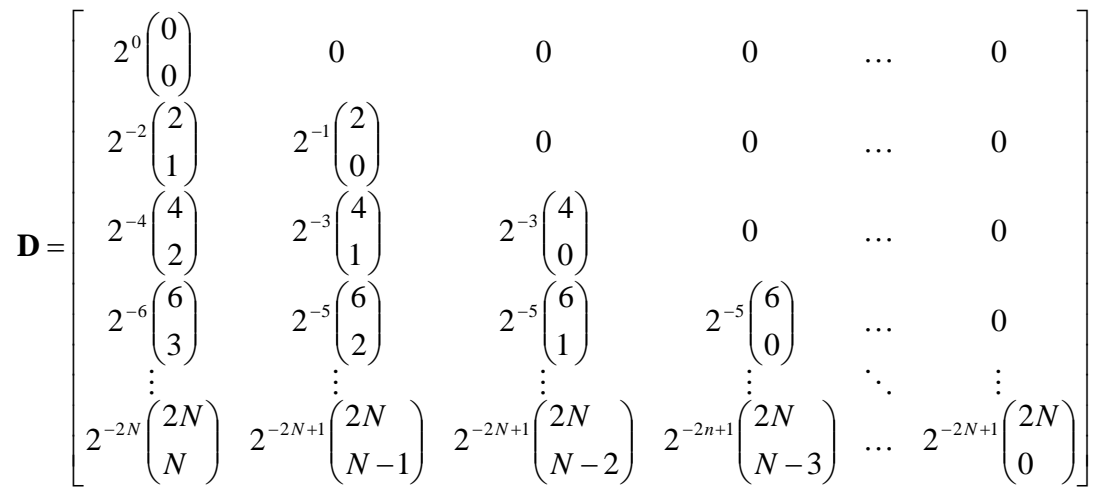

Then, by taking into account (7) we obtain

$$
\mathbf{T}^{*}(t)=\mathbf{X}(t)\left(\mathbf{D}^{-1}\right)^{T}
$$

and

$$
\left(\mathbf{T}^{*}(t)\right)^{(k)}=\mathbf{X}^{(k)}(t)\left(\mathbf{D}^{-1}\right)^{T}, \quad k=0,1,2
$$

To obtain the matrix $\mathbf{X}^{(k)}(t)$ in terms of the matrix $\mathbf{X}(t)$, we can use the following relation:

$$
\mathbf{X}^{(1)}(t)=\mathbf{X}(t) \mathbf{B}^{T}
$$

$$
\mathbf{X}^{(2)}(t)=\mathbf{X}^{(1)}(t) \mathbf{B}^{T}=\mathbf{X}(t)\left(\mathbf{B}^{T}\right)^{2}
$$

where

$$
\mathbf{B}=\left[\begin{array}{ccccc}
0 & 0 & 0 & \ldots & 0 \\
1 & 0 & 0 & \ldots & 0 \\
0 & 2 & 0 & \ldots & 0 \\
\ldots & \ldots & \ldots & \ldots & \ldots \\
0 & 0 & 0 & N & 0
\end{array}\right]
$$

Consequently, by substituting the matrix forms (11) and (12) into (10) we have the matrix relation 
$y_{N}^{(k)}=\mathbf{X}(t) \mathbf{B}^{k}\left(\mathbf{D}^{T}\right)^{-1} \mathbf{A}, k=0,1,2$

Moreover, since

$$
\mathbf{Y}^{m}=\mathbf{Y}^{m-1} \mathbf{Y}
$$

where

and

$$
\mathbf{Y}^{m-1}(x)=\left[\begin{array}{c}
y^{m-1}(x) \\
y^{m-1}(x) \\
\vdots \\
y^{m-1}(x)
\end{array}\right], \quad \overline{\mathbf{Y}}(x)=\left[\begin{array}{cccc}
y(x) & 0 & \cdots & 0 \\
0 & y(x) & \cdots & 0 \\
\vdots & \vdots & \ddots & \vdots \\
0 & 0 & \cdots & y(x)
\end{array}\right]
$$

$$
\overline{\mathbf{Y}}=\overline{\mathbf{T}} \overline{\mathbf{A}}
$$

where

$$
\overline{\mathbf{T}}(x)=\left[\begin{array}{cccc}
\mathbf{T}(x) & 0 & \cdots & 0 \\
0 & \mathbf{T}(x) & \cdots & 0 \\
\vdots & \vdots & \ddots & \vdots \\
0 & 0 & \cdots & \mathbf{T}(x)
\end{array}\right], \overline{\mathbf{A}}=\left[\begin{array}{cccc}
\mathbf{A} & 0 & \cdots & 0 \\
0 & \mathbf{A} & \cdots & 0 \\
\vdots & \vdots & \ddots & \vdots \\
0 & 0 & \cdots & \mathbf{A}
\end{array}\right]
$$

then we construct the following relation

$$
y_{N}^{2}\left(t_{i}\right)=y_{N}\left(t_{i}\right) y_{N}\left(t_{i}\right)=(\overline{\mathbf{T}} \overline{\mathbf{A}}) \mathbf{X}\left(t_{i}\right)\left(\mathbf{D}^{T}\right)^{-1} \mathbf{A}
$$

We can easily check the accuracy of the method. Since the truncated shifted Chebyshev series (3) is an approximate solution of the logistic models, when the solution $y_{N}(x)$ and its derivatives are substituted in models, the resulting equation must be satisfied approximately; that is, for $x=x_{q} \in[a, b], q=0,1,2, \ldots$

$$
E\left(x_{q}\right)=\left|\frac{d y_{N}}{d x}-r y_{N}\left(1-\frac{y_{N}}{K}\right)\right| \cong 0
$$

\section{Numerical solution of the models}

In this section, to show the efficiency of proposed method exampleas are given. Here after comparision between proposed method and other methods are presented.

\section{Example 1}

In this example we solve the

$$
\frac{d y}{d t}=y(1-y)
$$


Logistic growth model by using Chebyshev expansion method with the initial condition $y(0)=2[7]$. We seek approximate solutions in the form

$$
y_{N}(t)=\sum_{n=0}^{N} a_{n} T_{n}^{*}(t)
$$

Then, we get the matrix relation for Eq.(2)

$$
\left(\mathbf{X B}\left(\mathbf{D}^{T}\right)^{-1}-\mathbf{X}\left(\mathbf{D}^{T}\right)^{-1}+(\overline{\mathbf{T}} \overline{\mathbf{A}}) \mathbf{X}\left(\mathbf{D}^{T}\right)^{-1}\right) \mathbf{A}=\mathbf{F}
$$

and the initial condition of matrix representation

$$
y_{N}(0)=\mathbf{X}(0)\left(\mathbf{D}^{T}\right)^{-1} \mathbf{A}=\left[\begin{array}{llll}
u_{10} & u_{11} & \cdots & u_{1 N}
\end{array}\right] \mathbf{A}=2
$$

where

$$
\mathbf{X}=\left[\begin{array}{ccccc}
1 & x_{0} & x_{0}{ }^{2} & \cdots & x_{0}{ }^{N} \\
1 & x_{1} & x_{1}{ }^{2} & \cdots & x_{1}{ }^{N} \\
1 & x_{2} & x_{2}{ }^{2} & \cdots & x_{2}{ }^{N} \\
\vdots & \vdots & \vdots & \ddots & \vdots \\
1 & x_{N} & x_{N}{ }^{2} & \cdots & x_{N}{ }^{N}
\end{array}\right] \quad \mathbf{F}=\left[\begin{array}{c}
0 \\
0 \\
0 \\
\vdots \\
0
\end{array}\right] \quad \overline{\mathbf{T}}=\left[\begin{array}{ccccc}
\mathbf{T}\left(x_{0}\right) & 0 & 0 & \cdots & 0 \\
0 & \mathbf{T}\left(x_{1}\right) & 0 & \cdots & 0 \\
0 & 0 & \mathbf{T}\left(x_{2}\right) & \cdots & 0 \\
\vdots & \vdots & \vdots & \ddots & \vdots \\
0 & 0 & 0 & \cdots & \mathbf{T}\left(x_{N}\right)
\end{array}\right]
$$

Hence, the fundamental matrix equation (19) corresponding to Eq. (2) can be written in the form

$$
\mathbf{W A}=\mathbf{0} \text { or }[\mathbf{W} ; \mathbf{F}], \quad \mathbf{W}=\left[w_{i, j}\right], i, j=0,1, \ldots, N
$$

where

$$
\mathbf{W}=\mathbf{X B}\left(\mathbf{D}^{T}\right)^{-1}-\mathbf{X}\left(\mathbf{D}^{T}\right)^{-1}+(\overline{\mathbf{T}} \overline{\mathbf{A}}) \mathbf{X}\left(\mathbf{D}^{T}\right)^{-1}
$$

To obtain the solution of Eq. (2) under the initial conditions, by replacing the row matrices (20) by the last 1 rows of the matrix (21), we have the new augmented matrix,

$$
[\tilde{\mathbf{W}} ; \tilde{\mathbf{F}}]=\left[\begin{array}{cccccc}
w_{00} & w_{01} & \cdots & w_{0 N} & ; & 0 \\
w_{10} & w_{11} & \cdots & w_{1 N} & ; & 0 \\
\vdots & \vdots & \ddots & \vdots & \vdots & \vdots \\
w_{N-20} & w_{N-21} & \cdots & w_{N-2 N} & \vdots & 0 \\
w_{N-10} & w_{N-11} & \cdots & w_{N-1 N} & ; & 0 \\
u_{10} & u_{11} & \cdots & u_{1 N} & ; & 2
\end{array}\right]
$$

So, we obtained to a system of $(N+1)$ nonlinear algebraic equations with unknown shifted Chebyshev coefficients. Thus, we obtain the Chebyshev polynomial solution. For $N=5, N=6$ and $N=7$, we give the numerical results in Table 1 and plotted the numerical results in Fig.1-2. We compare the numerical results Homotopy perturbation 
method [9], Adomian decomposition method [6] and Present method in Table 2. Here, all computations are performed in the Maple 13 and all graphics are drawn by Matlab.

Table 1: Numerical result for Example 1

\begin{tabular}{cccccccc}
\hline $\mathrm{X}$ & Exact & & \multicolumn{5}{c}{ Present Method } \\
& Solution & $\mathrm{N}=5$ & $\mathrm{~N}_{\mathrm{e}}=5$ & $\mathrm{~N}=6$ & $\mathrm{~N}_{\mathrm{e}}=6$ & $\mathrm{~N}=7$ & $\mathrm{~N}_{\mathrm{e}}=7$ \\
\hline 0.0 & 2.000000 & 2.000000 & $0.000 \mathrm{E}-0$ & 2.000000 & $0.100 \mathrm{E}-8$ & 2.000000 & $0.000 \mathrm{E}-0$ \\
0.2 & 1.693094 & 1.692985 & $0.108 \mathrm{E}-3$ & 1.693110 & $0.161 \mathrm{E}-4$ & 1.693102 & $0.88 \mathrm{E}-5$ \\
0.4 & 1.504121 & 1.504343 & $0.222 \mathrm{E}-3$ & 1.504094 & $0.271 \mathrm{E}-4$ & 1.504107 & $0.141 \mathrm{E}-4$ \\
0.6 & 1.378180 & 1.377784 & $0.396 \mathrm{E}-3$ & 1.378183 & $0.309 \mathrm{E}-5$ & 1.378197 & $0.170 \mathrm{E}-4$ \\
0.8 & 1.289764 & 1.290240 & $0.476 \mathrm{E}-3$ & 1.289759 & $0.501 \mathrm{E}-5$ & 1.289742 & $0.212 \mathrm{E}-4$ \\
1.0 & 1.225399 & 1.221358 & $0.404 \mathrm{E}-2$ & 1.226409 & $0.100 \mathrm{E}-2$ & 1.225150 & $0.249 \mathrm{E}-3$ \\
\hline
\end{tabular}

Table 2: Comparision of numerical result for Example 1

\begin{tabular}{|c|c|c|c|}
\hline$X$ & Present method & $\begin{array}{c}\text { Homtopy } \\
\text { per. method[9] }\end{array}$ & $\begin{array}{c}\text { Adomian } \\
\text { decomp. method[6] }\end{array}$ \\
\hline 0.0 & 2.000000000 & 2.0000000000 & 2.0000000000 \\
\hline 0.2 & 1.6931102983 & 1.6932805333 & 1.6924480000 \\
\hline 0.4 & 1.5040941842 & 1.5236181333 & 1.4703360000 \\
\hline 0.6 & 1.3781839318 & 1.6597808000 & 1.0528640000 \\
\hline 0.8 & 1.2897591977 & 3.1168085333 & -0.293248000 \\
\hline 1.0 & 1.2264095932 & 8.9083333333 & -4.100000000 \\
\hline
\end{tabular}

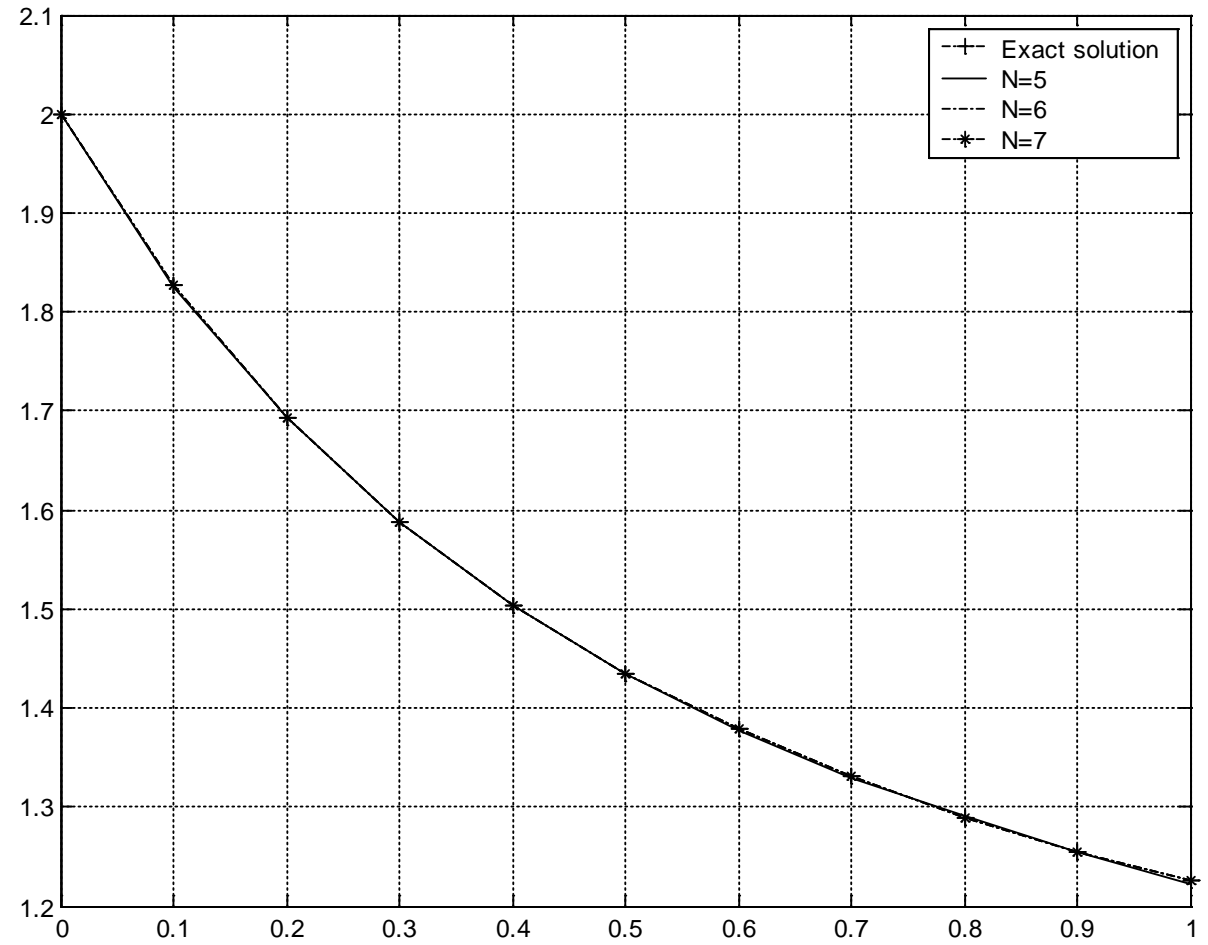

Fig. 1: Comparison numerical results and exact solution 


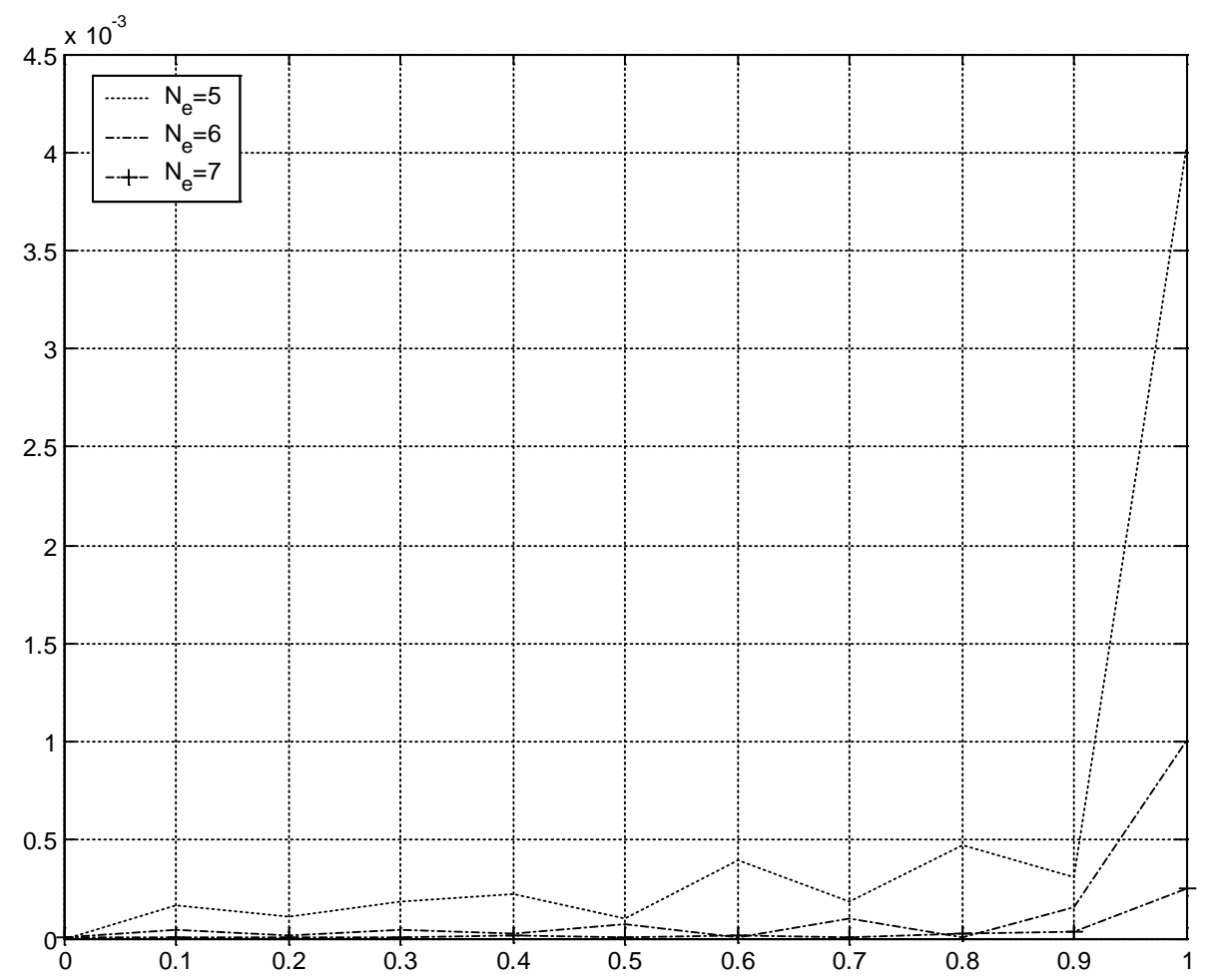

Fig. 2: Error function of logistic growth equation for various $N$

\section{Example 2}

In this example we investigate numerical solution of the predator-prey model which is defined before as

$$
\begin{aligned}
& \frac{d N}{d t}=N(a-b P) \\
& \frac{d P}{d t}=P(c N-d)
\end{aligned}
$$

We assume that the solutions can be written as truncated Chebyshev sums

$$
x(t)=\sum_{r=0}^{N} a_{r}^{1} T_{r}^{*}(t), \quad y(t)=\sum_{r=0}^{N} a_{r}^{2} T_{r}^{*}(t)
$$

then, matrix relation of solutions

$$
x(t)=\mathbf{T}(t) \mathbf{A}_{1}, y(t)=\mathbf{T}(t) \mathbf{A}_{2}
$$

where

$$
\begin{gathered}
\mathbf{T}(t)=\left[T_{0}^{*}(t) T_{1}^{*}(t) \ldots T_{N}^{*}(t)\right] \\
\mathbf{A}_{\mathbf{1}}=\left[\begin{array}{llll}
a_{0}^{1} & a_{1}^{1} \ldots & a_{N}^{1}
\end{array}\right]^{T} \quad \mathbf{A}_{2}=\left[\begin{array}{lll}
a_{0}^{2} & a_{1}^{2} \ldots & a_{N}^{2}
\end{array}\right]^{T} .
\end{gathered}
$$

Consequently, we get the approximate solution and its first-derivative of the matrix relations 


$$
\begin{aligned}
& x(t)=\mathbf{X}(t)\left(\mathbf{D}^{T}\right)^{-1} \mathbf{A}_{1} \quad \text { and } \quad x^{\prime}(t)=\mathbf{X}(t) \mathbf{B}^{T}\left(\mathbf{D}^{T}\right)^{-1} \mathbf{A}_{1} \\
& y(t)=\mathbf{X}(t)\left(\mathbf{D}^{T}\right)^{-1} \mathbf{A}_{2} \quad \text { and } \quad y^{\prime}(t)=\mathbf{X}(t) \mathbf{B}^{T}\left(\mathbf{D}^{T}\right)^{-1} \mathbf{A}_{2}
\end{aligned}
$$

The matrix relations (24) are written in Eqs.(4), we get

$$
\begin{aligned}
& \mathbf{X}(t) \mathbf{B}^{T}\left(\mathbf{D}^{T}\right)^{-1} \mathbf{A}_{1}+-a \mathbf{X}(t)\left(\mathbf{D}^{T}\right)^{-1} \mathbf{A}_{1}+b\left(\mathbf{T}(t) \mathbf{A}_{2}\right) \mathbf{X}(t)\left(\mathbf{D}^{T}\right)^{-1} \mathbf{A}_{1}=0 \\
& \mathbf{X}(t) \mathbf{B}^{T}\left(\mathbf{D}^{T}\right)^{-1} \mathbf{A}_{2}+c \mathbf{X}(t)\left(\mathbf{D}^{T}\right)^{-1} \mathbf{A}_{2}-d\left(\mathbf{T}(t) \mathbf{A}_{1}\right) \mathbf{X}(t)\left(\mathbf{D}^{T}\right)^{-1} \mathbf{A}_{2}=0
\end{aligned}
$$

The matrix form of (25)

$$
\left(\overline{\mathbf{Y}(t)} \overline{\mathbf{B}^{T}} \overline{\left(\mathbf{D}^{T}\right)^{-1}}+\mathbf{P}_{0} \overline{\mathbf{Y}(t)} \overline{\left(\mathbf{D}^{T}\right)^{-1}}+\mathbf{P}_{1} \overline{\mathbf{T}(t)} \overline{\mathbf{Y}(t)} \overline{\left(\mathbf{D}^{T}\right)^{-1}}\right) \mathbf{A}=\mathbf{F}
$$

where

$$
\begin{gathered}
\overline{\mathbf{Y}(t)}=\left[\begin{array}{cc}
\mathbf{Y}(t) & 0 \\
0 & \mathbf{Y}(t)
\end{array}\right] \overline{\mathbf{B}^{T}}=\left[\begin{array}{cc}
\mathbf{B}^{T} & 0 \\
0 & \mathbf{B}^{T}
\end{array}\right]\left(\mathbf{D}^{T}\right)^{-1}=\left[\begin{array}{cc}
\left(\mathbf{D}^{T}\right)^{-1} & 0 \\
0 & \left(\mathbf{D}^{T}\right)^{-1}
\end{array}\right] \\
\overline{\mathbf{T}(t)}=\left[\begin{array}{cc}
\mathbf{T}(t) \mathbf{A}_{2} & 0 \\
0 & \mathbf{T}(t) \mathbf{A}_{1}
\end{array}\right] \mathbf{P}_{0}=\left[\begin{array}{cc}
-a & 0 \\
0 & c
\end{array}\right] \mathbf{P}_{1}=\left[\begin{array}{cc}
b & 0 \\
0 & -d
\end{array}\right] \mathbf{F}=\left[\begin{array}{l}
0 \\
0
\end{array}\right] \mathbf{A}=\left[\begin{array}{l}
\mathbf{A}_{1} \\
\mathbf{A}_{1}
\end{array}\right]
\end{gathered}
$$

Using the shifted Chebyshev zeroes (8), we put of them in above matrix relation. We obtained

$$
\left.\left.\left(\overline{\mathbf{Y}\left(t_{i}\right)} \overline{\mathbf{B}^{T}} \overline{\left(\mathbf{D}^{T}\right)^{-1}}+\mathbf{P}_{0} \overline{\mathbf{Y}\left(t_{i}\right.}\right) \overline{\left(\mathbf{D}^{T}\right)^{-1}}+\mathbf{P}_{1} \overline{\mathbf{T}\left(t_{i}\right)} \overline{\mathbf{Y}\left(t_{i}\right.}\right) \overline{\left(\mathbf{D}^{T}\right)^{-1}}\right) \mathbf{A}=\mathbf{F}
$$

and

$$
\left(\overline{\overline{\mathbf{Y}}} \overline{\mathbf{B}^{T}}\left(\overline{\left.\mathbf{D}^{T}\right)^{-1}}+\overline{\mathbf{P}_{0}} \overline{\overline{\mathbf{Y}}} \overline{\left(\mathbf{D}^{T}\right)^{-1}}+\overline{\mathbf{P}_{1}} \overline{\mathbf{T}} \overline{\overline{\mathbf{Y}}} \overline{\left(\mathbf{D}^{T}\right)^{-1}}\right) \mathbf{A}=\overline{\mathbf{F}}\right.
$$

where

$$
\begin{aligned}
& \overline{\mathbf{Y}}(t)=\left[\begin{array}{ccc}
\mathbf{Y}(t) & \cdots & 0 \\
\vdots & \ddots & \vdots \\
0 & \cdots & \mathbf{Y}(t)
\end{array}\right] \overline{\mathbf{Y}}(t)=\left[\begin{array}{ccc}
\overline{\mathbf{Y}\left(t_{0}\right)} & \cdots & 0 \\
\vdots & \ddots & \vdots \\
0 & \cdots & \overline{\mathbf{Y}\left(t_{N}\right)}
\end{array}\right] \overline{\mathbf{T}}=\left[\begin{array}{ccc}
\overline{\mathbf{T}\left(t_{0}\right)} & \cdots & 0 \\
\vdots & \ddots & \vdots \\
0 & \cdots & \overline{\mathbf{T}\left(t_{N}\right)}
\end{array}\right] \\
& \overline{\mathbf{P}_{0}}=\left[\begin{array}{ccc}
\mathbf{P}_{0} & \cdots & 0 \\
\vdots & \ddots & \vdots \\
0 & \cdots & \mathbf{P}_{0}
\end{array}\right] \overline{\mathbf{P}_{1}}=\left[\begin{array}{ccc}
\mathbf{P}_{1} & \cdots & 0 \\
\vdots & \ddots & \vdots \\
0 & \cdots & \mathbf{P}_{1}
\end{array}\right] \overline{\mathbf{F}}=\left[\begin{array}{c}
\mathbf{F} \\
\vdots \\
\mathbf{F}
\end{array}\right]
\end{aligned}
$$

On the other hand, the matrix form for conditions can be written as

$$
\begin{aligned}
& x(0)=\mathbf{X}(0)\left(\mathbf{D}^{T}\right)^{-1} \mathbf{A}_{1} \equiv\left[\begin{array}{llll}
u_{00} & u_{01} & \cdots & u_{0 N}
\end{array}\right]=[\alpha] \\
& y(0)=\mathbf{X}(0)\left(\mathbf{D}^{T}\right)^{-1} \mathbf{A}_{2} \equiv\left[\begin{array}{llll}
u_{10} & u_{11} & \cdots & u_{1 N}
\end{array}\right]=[\beta]
\end{aligned}
$$


Hence, the fundamental matrix equation corresponding to Eq. (4) can be written in the form

$\mathbf{W A}=\mathbf{F}$ or $[\mathbf{W} ; \mathbf{F}], \quad \mathbf{W}=\left[w_{i, j}\right], i, j=0,1, \ldots, N$

where

$$
\mathbf{W}=\overline{\overline{\mathbf{Y}}} \overline{\mathbf{B}^{T}}\left(\overline{\left.\mathbf{D}^{T}\right)^{-1}}+\overline{\mathbf{P}_{0}} \overline{\overline{\mathbf{Y}}} \overline{\left(\mathbf{D}^{T}\right)^{-1}}+\overline{\mathbf{P}_{1}} \overline{\mathbf{T}} \overline{\overline{\mathbf{Y}}} \overline{\left(\mathbf{D}^{T}\right)^{-1}}\right.
$$

To obtain the solution of Eq. (4) under conditions, by replacing the row matrices (26ab) by the last 2 rows of the matrix (27), we have the new augmented matrix,

and then

$$
\tilde{\mathbf{W}}=\left[\begin{array}{cccc}
w_{00} & w_{01} & \cdots & w_{0(2 N+2)} \\
w_{10} & w_{11} & \cdots & w_{1(2 N+2)} \\
\vdots & \vdots & \ddots & \vdots \\
w_{(2 N) 0} & w_{(2 N) 1} & \cdots & w_{(2 N)(2 N+2)} \\
u_{00} & u_{01} & \cdots & u_{0(2 N+2)} \\
u_{10} & u_{11} & \cdots & u_{1(2 N+2)}
\end{array}\right]
$$

$$
\tilde{\mathbf{W A}}=\mathbf{F}
$$

where

$$
F=\left[\begin{array}{c}
\mathbf{0} \\
\mathbf{0} \\
\vdots \\
\mathbf{G}
\end{array}\right] \quad \mathbf{G}=\left[\begin{array}{c}
\alpha \\
\beta
\end{array}\right]
$$

So we obtained to a system of $2(N+1) \times 2(N+1)$ nonlinear algebraic equations with unknown shifted Chebyshev coefficients. We solved the Eq.(28) by using mathematical program Maple 13 and graphics are drawn with Matlab.

For numerical study the following values $\alpha=14, \beta=18, a=0.1, b=1, c=1$ and $d=1$ are used [4]. We seek the approximate solutions by truncated shifted Chebyshev series

$$
x(t)=\sum_{r=0}^{N} a_{r}^{1} T_{r}^{*}(t), \quad y(t)=\sum_{r=0}^{N} a_{r}^{2} T_{r}^{*}(t)
$$

where we assume $N=3$. Then, the zeroes of the $T_{4}{ }^{*}(t)$ are

$$
t_{0}=\frac{1}{2}-\frac{1}{2} \cos \left(\frac{\pi}{8}\right), t_{1}=\frac{1}{2}-\frac{1}{2} \cos \left(\frac{3 \pi}{8}\right), t_{2}=\frac{1}{2}+\frac{1}{2} \cos \left(\frac{3 \pi}{8}\right), t_{3}=\frac{1}{2}+\frac{1}{2} \cos \left(\frac{\pi}{8}\right)
$$

Hence

$$
\begin{aligned}
& \mathbf{Y}\left(t_{0}\right)=\left[\begin{array}{llll}
1 & 0.038060 & 0.001448 & 0.000055
\end{array}\right] \\
& \mathbf{Y}\left(t_{1}\right)=\left[\begin{array}{llll}
1 & 0.308658 & 0.095269 & 0.029405
\end{array}\right]
\end{aligned}
$$




$$
\begin{aligned}
& \mathbf{Y}\left(t_{2}\right)=\left[\begin{array}{llll}
1 & 0.691341 & 0.477953 & 0.330429
\end{array}\right] \\
& \mathbf{Y}\left(t_{3}\right)=\left[\begin{array}{llll}
1 & 0.961939 & 0.925328 & 0.890109
\end{array}\right] \\
& \mathbf{B}^{T}=\left[\begin{array}{llll}
0 & 1 & 0 & 0 \\
0 & 0 & 2 & 0 \\
0 & 0 & 0 & 3 \\
0 & 0 & 0 & 0
\end{array}\right] \quad\left(\mathbf{D}^{T}\right)^{-1}=\left[\begin{array}{cccc}
1 & -1 & 1 & -1 \\
0 & 2 & -8 & 18 \\
0 & 0 & 8 & -48 \\
0 & 0 & 0 & 32
\end{array}\right] \\
& \mathbf{T}\left(t_{0}\right) \mathbf{A}_{1}=a_{0}^{1}-0.923879 a_{1}^{1}+0.707106 a_{2}^{1}-0.382683 a_{3}^{1} \\
& \mathbf{T}\left(t_{1}\right) \mathbf{A}_{1}=a_{0}^{1}-382683 a_{1}^{1}-0.707106 a_{2}^{1}+0.923879 a_{3}^{1} \\
& \mathbf{T}\left(t_{2}\right) \mathbf{A}_{1}=a_{0}^{1}+382683 a_{1}^{1}-0.707106 a_{2}^{1}-0.923879 a_{3}^{1} \\
& \mathbf{T}\left(t_{3}\right) \mathbf{A}_{1}=a_{0}^{1}+923879 a_{1}^{1}+0.7071066 a_{2}^{1}+0.382683 a_{3}^{1} \\
& \mathbf{T}\left(t_{0}\right) \mathbf{A}_{2}=a_{0}^{2}-0.923879 a_{1}^{2}+0.707106 a_{2}^{2}-0.382683 a_{3}^{2} \\
& \mathbf{T}\left(t_{1}\right) \mathbf{A}_{2}=a_{0}^{2}-382683 a_{1}^{2}-0.707106 a_{2}^{2}+0.923879 a_{3}^{2} \\
& \mathbf{T}\left(t_{2}\right) \mathbf{A}_{2}=a_{0}^{2}+382683 a_{1}^{2}-0.707106 a_{2}^{2}-0.923879 a_{3}^{2} \\
& \mathbf{T}\left(t_{3}\right) \mathbf{A}_{3}=a_{0}^{2}+923879 a_{1}^{2}+0.7071066 a_{2}^{2}+0.382683 a_{3}^{2}
\end{aligned}
$$

with conditions

$$
\left[\begin{array}{cccccccccc}
1 & -1 & 1 & -1 & 0 & 0 & 0 & 0 & ; & 14 \\
0 & 0 & 0 & 0 & 1 & -1 & 1 & -1 & ; & 18
\end{array}\right]
$$

These matrix are substituting in Eq.(28), we obtained to a system of $2(n+1) \times 2(n+1)$ nonlinear algebraic equations. This nonlinear sytem is solved, we gained the shifted Chebyshev coefficients,

$$
\mathbf{A}_{1}=\left[\begin{array}{c}
2.618855 \\
-5.102665 \\
4.571254 \\
-1.707224
\end{array}\right] \quad \mathbf{A}_{2}=\left[\begin{array}{c}
18.334923 \\
-4.934231 \\
-3.544386 \\
1.724768
\end{array}\right]
$$

We put the these coefficients in Eq.(28), we obtain the approximate solutions for $N=3$

$$
\begin{aligned}
& x(t)=14-77.505413 t+118.516822 t^{2}-54.631188 t^{3} \\
& y(t)=18+49.532465 t-111.143992 t^{2}+55.192600 t^{3}
\end{aligned}
$$

For $N=4$, approximate solution is obtained

$$
\begin{aligned}
& x(t)=14-250.6 t t+1424.618550 t^{2}-2840.335841 t^{3}+1812.627521 t^{4} \\
& y(t)=18-234 t-1490.402822 t^{2}+2959.652578 t^{3}-1858.600392 t^{4}
\end{aligned}
$$

For $N=5$, approximate solution is obtained 


$$
\begin{aligned}
& x(t)=14+365.989241 t-1798.989241 t^{2}+3413.533114 t^{3}-2629.755264 t^{4}+647.350767 t^{5} \\
& y(t)=18-365.98924 t+1859.983387 t^{2}-3636.520042 t^{3}+2925.596518 t^{4}-778.890081 t^{5}
\end{aligned}
$$

These results are plotted in Figs. 3-5.

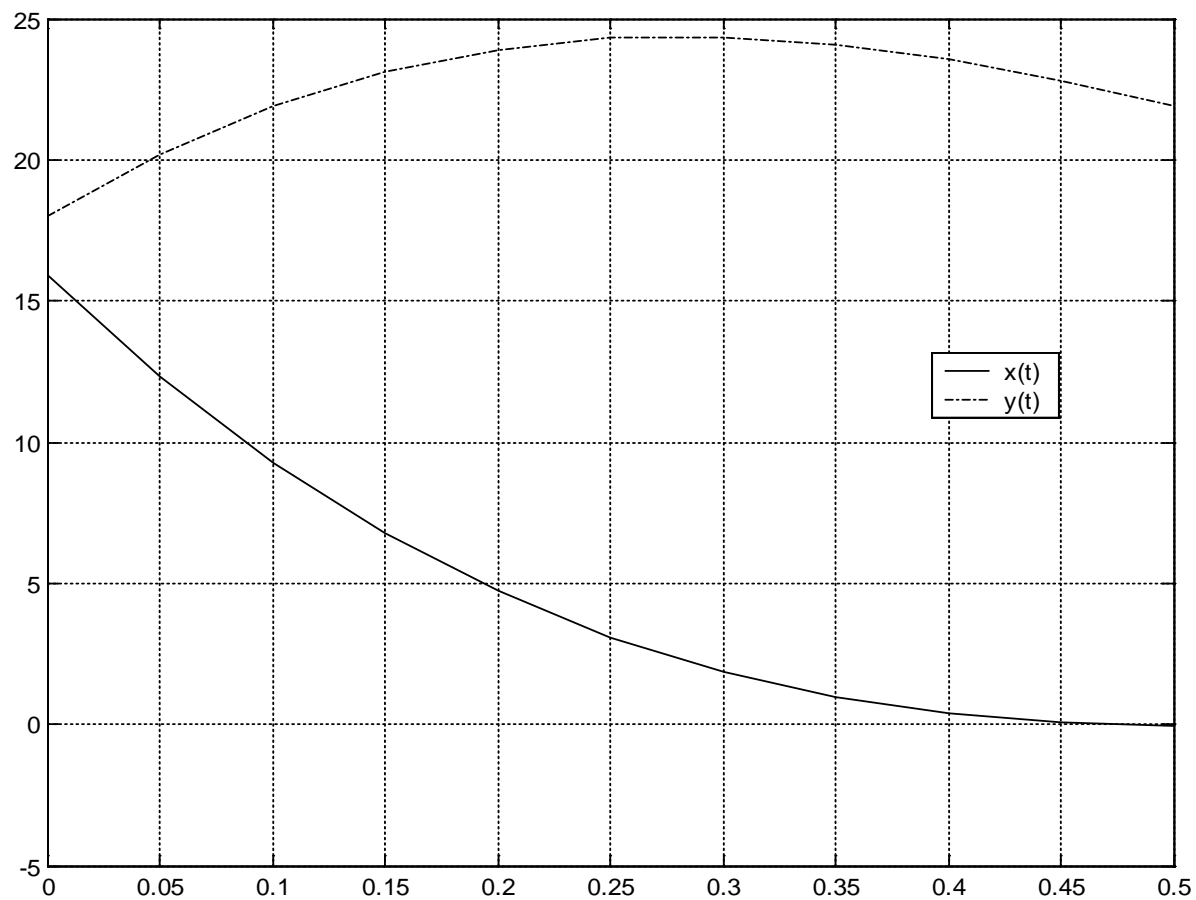

Fig.3. Numbers of the rabbits and foxes versus time for $N=3$

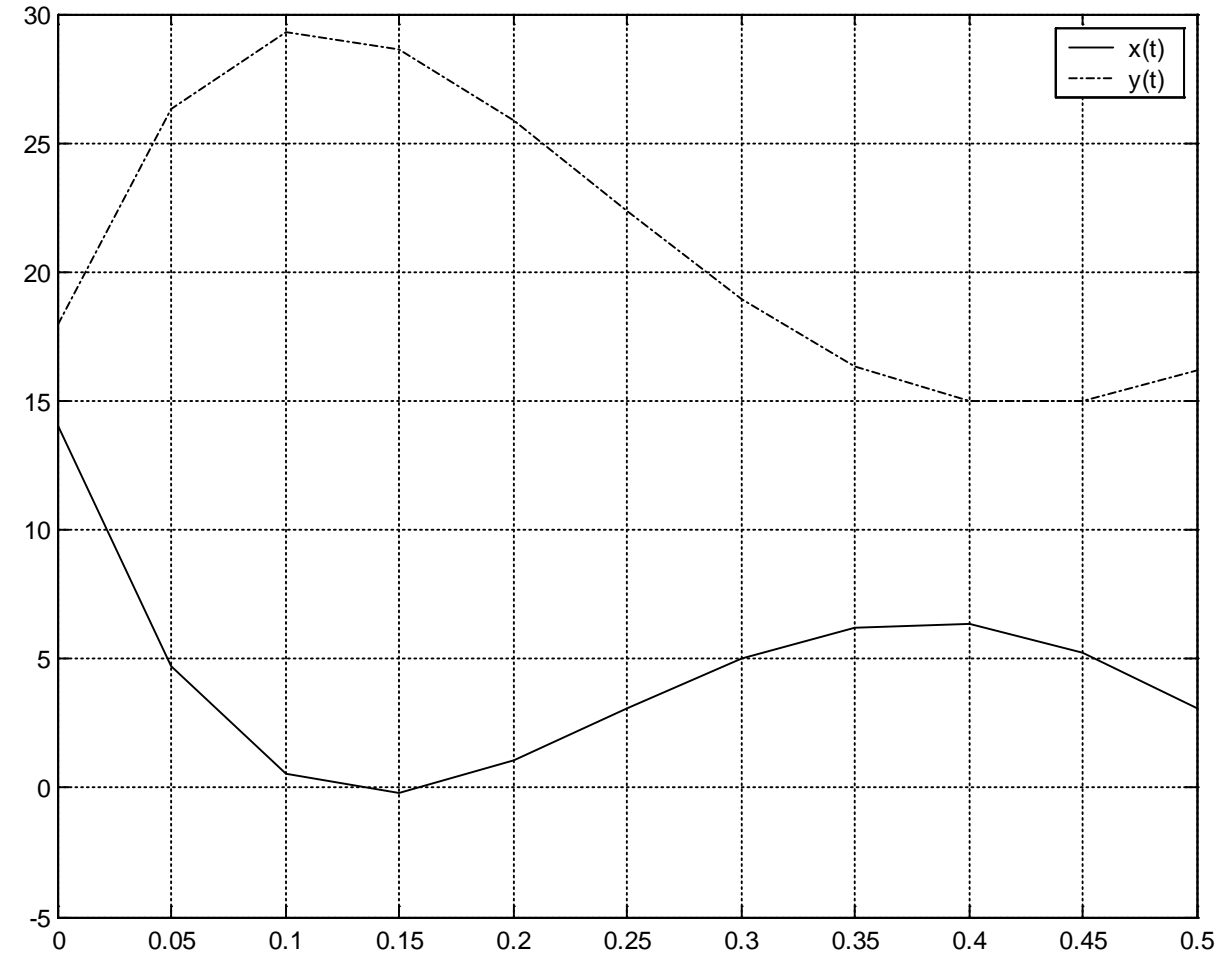

Fig.4. Numbers of the rabbits and foxes versus time for $N=4$ 


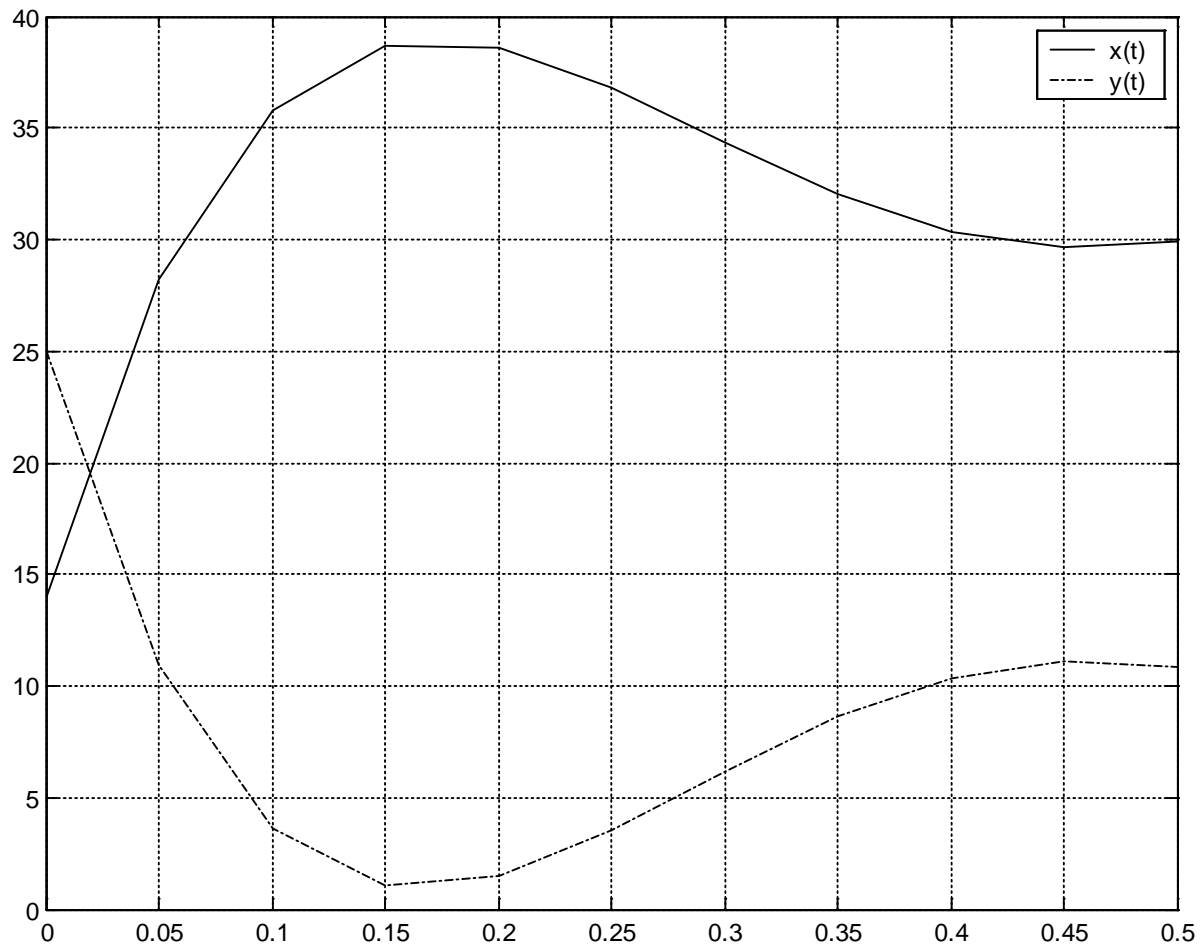

Fig.5. Numbers of the rabbits and foxes versus time for $N=5$

\section{Example 3}

In this example we consider numerical solution of the 2-species Lotka-Volterra competition model

$$
\begin{aligned}
& \frac{d u}{d t}=u(1-u-a v) \\
& \frac{d v}{d t}=\rho v(1-v-b u)
\end{aligned}
$$

Let assume that the solutions of equation can be written as truncated Chebyshev sums

$u(t)=\sum_{r=0}^{N} a_{r}^{1} T_{r}^{*}(t), \quad v(t)=\sum_{r=0}^{N} a_{r}^{2} T_{r}^{*}(t)$

then, matrix relation of solutions

$u(t)=\mathbf{T}(t) \mathbf{A}_{1}, v(t)=\mathbf{T}(t) \mathbf{A}_{2}$

where

$$
\begin{gathered}
\mathbf{T}(t)=\left[T_{0}^{*}(t) T_{1}^{*}(t) \ldots T_{N}^{*}(t)\right] \\
\mathbf{A}_{\mathbf{1}}=\left[\begin{array}{llll}
a_{0}^{1} & a_{1}^{1} \ldots & a_{N}^{1}
\end{array}\right]^{T} \quad \mathbf{A}_{2}=\left[\begin{array}{lll}
a_{0}^{2} & a_{1}^{2} \ldots a_{N}^{2}
\end{array}\right]^{T} .
\end{gathered}
$$

Consequently, we get the approximate solution and its first-derivative of the matrix relations 


$$
\begin{aligned}
& u(t)=\mathbf{X}(t)\left(\mathbf{D}^{T}\right)^{-1} \mathbf{A}_{1} \quad \text { and } \quad u^{\prime}(t)=\mathbf{X}(t) \mathbf{B}^{T}\left(\mathbf{D}^{T}\right)^{-1} \mathbf{A}_{1} \\
& v(t)=\mathbf{X}(t)\left(\mathbf{D}^{T}\right)^{-1} \mathbf{A}_{2} \quad v^{\prime}(t)=\mathbf{X}(t) \mathbf{B}^{T}\left(\mathbf{D}^{T}\right)^{-1} \mathbf{A}_{2}
\end{aligned}
$$

The matrix relation of Eq.(6) is written as

$$
\left[\begin{array}{c}
\mathbf{X}(t) \mathbf{B}^{T}\left(\mathbf{D}^{T}\right)^{-1}+a\left(\mathbf{T}(t) \mathbf{A}_{2}\right) \mathbf{X}(t)\left(\mathbf{D}^{T}\right)^{-1}+\left(\mathbf{T}(t) \mathbf{A}_{1}\right) \mathbf{X}(t)\left(\mathbf{D}^{T}\right)^{-1}-\mathbf{X}(t)\left(\mathbf{D}^{T}\right)^{-1} \\
\mathbf{X}(t) \mathbf{B}^{T}\left(\mathbf{D}^{T}\right)^{-1}+b \rho\left(\mathbf{T}(t) \mathbf{A}_{2}\right) \mathbf{X}(t)\left(\mathbf{D}^{T}\right)^{-1}+\rho\left(\mathbf{T}(t) \mathbf{A}_{1}\right) \mathbf{X}(t)\left(\mathbf{D}^{T}\right)^{-1}-\rho \mathbf{X}(t)\left(\mathbf{D}^{T}\right)^{-1}
\end{array}\right]\left[\begin{array}{l}
\mathbf{A}_{1} \\
\mathbf{A}_{2}
\end{array}\right]=\left[\begin{array}{l}
0 \\
0
\end{array}\right]
$$

We take $a=1, b=0.8$ and $\rho=1$ and the initial conditions $u(0)=1$ and $v(0)=1$ [6]. Similiarly, we apply the procedure in Section 3.2. We obtained to a system of $2(N+1) \times 2(N+1)$ nonlinear algebraic equations with unknown shifted Chebyshev coefficients. For $N=5$, we get the approximate solutions

$u(t)=0.999999-0.999999 t+1.467206 t^{2}-1.728703 t^{3}+1.302433 t^{4}-0.423262 t^{5}$ $v(t)=1-x+1.371353 t^{2}-1.580208 t^{3}+1.175924 t^{4}-0.379854 t^{5}$

We plotted these results in Fig. 6.

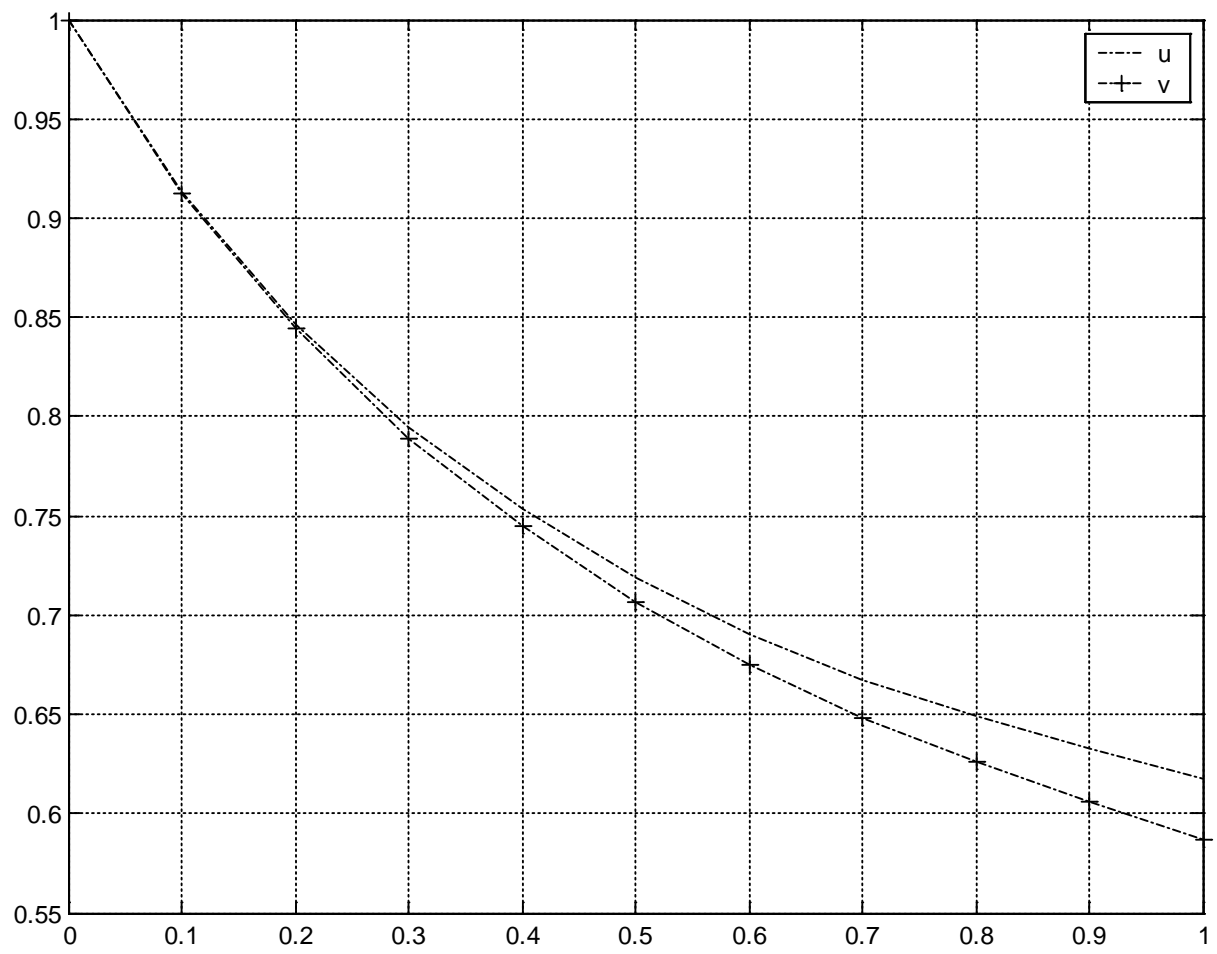

Fig 6: Numerical result of Present method for $N=5$

\section{Conclusion}

In this article, we gain the approximate solutions of the continuous population models for single and interacting species by Chebyshev expansion method. In the logistic growth model is successively solved by this method. In Table 1 , we see that when $\mathrm{N}$ is increasing, approximate solution is closed the exact solution and so absolute errors is 
decreasing. In the Lotka-Volterra model, as the plots Fig. 3-5 said the number of foxes increase, as the number of rabbits, the source of food for foxes, decrease. Foxes will reach their maximum as the rabbits reach their minimum. Finally, Fig. 6 is said that species are to be a positive function of others. Also the CPU time for A model is less then B model and CPU time for B model is less then C model.

\section{References}

[1] Murray, J.D., Mathematical Biology, Springer, Berlin, (1993).

[2] Simmons, G.F., Differential Equations with Applications and Historical Notes, McGraw-Hill, (1972).

[3] Biazar, J. ve Montazeri, R., A computational method for solution of the prey and predator problem, Applied Mathematics and Computation, 163,2,841-847, (2005).

[4] Biazar, J., Ilie, M. ve Khoshkenar,A., A new approach to the solution of the prey and predator problem and comparison of the results with the Adomian method, Applied Mathematics and Computation, 171,1,486-491, (2005).

[5] Rafei, M., Daniali, H., Ganji, D.D. ve Pashaedi, H., Solution of the prey and predator problem by homotopy perturbation method, Applied Mathematics and Computation, 188, 1419-1425, (2007).

[6] Pamuk, S., The decomposition method for continuous population models for single and interacting species, Applied Mathematics and Computation, 163, 79-88, (2005).

[7] Pamuk, S. ve Pamuk, N., He's homotopy perturbation method for continuous population models for single and interacting species, Computational Mathematics and Applications, 59, 612-621, (2010).

[8] Pamuk, S., A review of some recent results for the approximate analytical solutions of non-linear differential equations, Mathematical Problems in Engineering, 34, (2009).

[9] Pamuk, S. ve Pamuk, N., He's homotopy perturbation method for continuous population models for single and interacting species, Computational Mathematics and Applications, 59, 612-621, (2010).

[10] Hu, X., Liu, G. ve Yan, J., Existence of multiple positive periodic solutions of delayed predator-prey models with functional responses, Computational Mathematics and Applications, 52, 1453-1462, (2006).

[11] Edelstein-Keshet, L., Mathematical Models in Biology, Random House, New York, (1988).

[12] Takeuchi, Y., Du, N.H., Hieu, N.T. ve Sato, K., Evolution of predator-prey systems described by a Lotka-Volterra equation under random environment, Journal of Mathematical Analysis and Applications, 323, 938-957, (2006).

[13] Akyüz, A. ve Sezer, M., Chebyshev polynomial solutions of systems of highorder linear differential equations with variable coeficients, Applied Mathematics and Computation, 144,237-247, (2003).

[14] Gülsu, M., Öztürk, Y. ve Sezer, M., A new collocation method for solution of mixed linear integro-differential-difference equations, Applied Mathematics and Computation, 216, 2183-2198, (2010).

[15] Sezer, M. ve Dogan, S., Chebyshev series solutions of Fredholm integral equations, International Journal of Mathematical Education in Science and Technology, 27, 5, 649-657, (1996). 
[16] Gülsu, M., Öztürk, Y. ve Sezer, M., On the solution of the Abel equation of the second kind by the shifted Chebyshev polynomials, Applied Mathematics and Computation, 217, 4827-4833, (2011).

[17] Daşçığlu, A. ve Yaslan, H., The solution of high-order nonlinear ordinary differential equations by Chebyshev polynomials, Applied Mathematics and Computation, 217, 2, 5658-5666,(2011).

[18] Daşcioglu, A., Chebyshev solutions of systems of linear integral equations, Applied Mathematics and Computation, 151, 221-232, (2004).

[19] Dascioglu, A., ve Sezer, M., Chebyshev polynomial solutions of systems of higher-order linear Fredholm-Volterra integro-differential equations, Journal of The Franklin Institute, 342, 688-701, (2005).

[20] Öztürk, Y., Gülsu, A., ve Gülsu, M., On solution of a modified epidemiological model for drug release systems, Scholars Journal of Physics, Mathematics and Statistics, 3,1, 1-5, (2016).

[21] Öztürk, Y., Gülsu, A., ve Gülsu, M., A numerical approach for solving modified epidemiological model for drug release systems, Nevşehir Bilim ve Teknoloji Dergisi, 2 ,2, 56-64, (2013).

[22] Öztürk, Y., Gülsu, A.,ve Gülsu, M., A numerical method for solving the mathematical model of controlled drug release, Bitlis Eren Üniversitesi Fen Bilimleri Dergisi, 2, 2, 169-175, (2013).

[23] Mason, J.C. ve Handscomb, D.C., Chebyshev polynomials, Chapman and Hall/CRC, New York,(2003).

[24] Body, J.P., Chebyshev and fourier spectral methods, University of Michigan, New York, (2000).

[25] Rivlin, T. J., Introduction to the approximation of functions, London, (1969). 